\title{
Um estudo comparativo de classificadores na previsão da evasão de alunos em EAD
}

\author{
Jorge L. C. Ramos ${ }^{1}$, João C. Sedraz Silva ${ }^{1}$, Leonardo C. Prado ${ }^{1}$ Alex S. Gomes ${ }^{2}$, Rodrigo \\ L. Rodrigues ${ }^{3}$ \\ ${ }^{1}$ Universidade Federal do Vale do São Francisco - Juazeiro - BA - Brasil \\ ${ }^{2}$ Universidade Federal de Pernambuco - Recife-PE, Brasil \\ ${ }^{3}$ Universidade Federal Rural de Pernambuco - Recife-PE, Brasil.
}

\begin{abstract}
The use of machine learning and data mining algorithms in educational contexts has evolved due to the large availability of data generated mainly in virtual learning environments. This study makes a comparative analysis of five classifiers in the task of predicting students with risk of dropping out in undergraduate courses by distance education. The results showed a small advantage for the use of Logistic Regression in the data analyzed, with success rates above $90 \%$ in the predictive model.
\end{abstract}

Resumo. $O$ uso de algoritmos de mineração de dados e de aprendizagem de máquina em contextos educacionais tem evoluído em razão da grande disponibilidade de dados geradas principalmente em ambientes virtuais de aprendizagem. Este estudo faz uma análise comparativa de cinco classificadores na tarefa de predição de alunos com risco de evasão em cursos de graduação por EAD. Os resultados apontaram uma pequena vantagem para o uso da Regressão Logística nos dados analisados, com taxas de sucesso acima de $90 \%$ no modelo preditivo.

\section{Introdução}

A Educação a Distância (EAD) vem crescendo de maneira acentuada nos últimos anos e assumido um importante papel no cenário educacional. Essa modalidade viabiliza, dentre outras oportunidades, a formação em horário flexível distinto dos ambientes escolares presenciais, além de possuir um alcance geográfico abrangente, que permite a inclusão cada vez maior de pessoas na educação profissional e superior.

Os dados do crescimento da EAD no Brasil, fornecidos pelo Instituto Nacional de Estudos e Pesquisas Educacionais Anísio Teixeira (INEP) ${ }^{1}$, apontam um crescimento significativo da modalidade no país nos últimos anos. O INEP é um órgão federal vinculado ao Ministério da Educação (MEC) com o objetivo de promover estudos, pesquisas e avaliações sobre o Sistema Educacional Brasileiro. Segundo esses dados, o número de alunos na modalidade graduação a distância continua crescendo, atingindo 1,34 milhão em 2014, o que já representa uma participação de $17,1 \%$ do total de matrículas da educação superior. Enquanto o número de matrículas em cursos de graduação presenciais cresceu 5,4\% entre 2013 e 2014, na modalidade a distância, o aumento foi de $16,3 \%$.

Aliadas ao incremento da modalidade, as pesquisas na área também devem buscar atenuar ou resolver problemas que surgem decorrentes dessa expansão, como, por exemplo, os altos índices de evasão ainda verificados na modalidade. Para a maioria das instituições brasileiras que participam do Censo Anual da EAD no Brasil, o maior obstáculo enfrentado tem sido a evasão nos cursos, onde para $32 \%$ das instituições pesquisadas, a taxa média de evasão

${ }^{1}$ http://portal.inep.gov.br/ 
VII Congresso Brasileiro de Informática na Educação (CBIE 2018)

Anais do XXIX Simpósio Brasileiro de Informática na Educação (SBIE 2018)

em cursos totalmente a distância, em 2016, foi entre $11 \%$ e $25 \%$ e, para outras $13 \%$ das instituições, a taxa foi de $26 \%$ a $35 \%$ [ABED, 2017].

A EAD é desenvolvida, em sua maioria, por ambientes virtuais de ensino e aprendizagem (AVA), onde todo o conteúdo do curso é apresentado, assim como recursos de interação e avaliação são disponibilizados. Os dados extraídos desses ambientes virtuais podem indicar características comportamentais dos alunos e, com isso, permitir análises inferenciais e preditivas, a partir do uso de tecnologias como a de aprendizagem de máquina, usada neste trabalho.

Este trabalho apresenta uma análise comparativa de cinco classificadores usados em aprendizagem de máquina, avaliando o seu uso na definição de modelo preditivo da evasão dos alunos, a partir de um conjunto de dados de interação e da estrutura do ambiente virtual em uso por uma universidade pública, na oferta de dois cursos de graduação totalmente por EAD.

\section{Fundamentação Teórica}

\subsection{Aprendizagem de máquina}

A aprendizagem de máquina investiga como computadores podem aprender (ou melhorar o seu desempenho) com base em dados analisados. A principal área de pesquisa é desenvolver algoritmos para aprenderem automaticamente a reconhecer padrões complexos e tomar decisões inteligentes baseadas nos dados. Por exemplo, é um problema de aprendizado de máquina programar um computador para que ele possa reconhecer automaticamente códigos postais manuscritos no correio após a aprendizagem de um conjunto de exemplos [Han et al., 2011].

Para Lantz (2013), a aprendizagem de máquina é o campo de estudo interessado no desenvolvimento de algoritmos de computador para transformar dados em ação inteligente. Esse campo é originado em um ambiente na qual os dados estão disponíveis e os métodos estatísticos e poder de computação evoluíram rápida e simultaneamente. O crescimento de dados exigiu poder de computação adicional, que, por sua vez, impulsionou o desenvolvimento de métodos estatísticos para a análise de grandes conjuntos de dados. Isso criou um ciclo evolutivo, permitindo que os dados, ainda maiores e mais interessantes, possam ser coletados e analisados.

\subsection{Classificadores}

Classificação, em aprendizagem de máquina, é a tarefa de organizar objetos em uma entre diversas categorias pré-definidas. Nessa tarefa, o modelo analisa o conjunto de dados fornecidos, na qual cada dado já contém o rótulo, indicando a qual categoria ele pertence, a fim de "aprender" como classificar novos dados. Por exemplo, um programa de e-mail pode tentar classificar um e-mail como "legítimo" ou como "spam", usando a classificação baseada em emails anteriormente recebidos e rotulados [Han et al., 2011].

$\mathrm{Na}$ classificação, os algoritmos que implementam esse processo são chamados de classificadores. O termo "classificador", às vezes, também se refere à função matemática implementada por meio de um algoritmo, que mapeia os dados de entrada para uma categoria.

No processo de classificação, duas etapas principais são realizadas: (a) a aprendizagem ou treinamento, na qual dados de treinamento são analisados por um algoritmo classificador, em que são atribuídos os rótulos de classe e o modelo aprendido ou classificador é representado sob a forma de regras de classificação; e (b) a classificação, na qual os dados de teste são usados para estimar a acurácia das regras de classificação. Se a acurácia for considerada aceitável, as regras podem ser aplicadas para a classificação de novos dados [Han et al., 2011].

Os principais classificadores são agrupados em cinco categorias principais: árvores de decisão, classificadores baseados em regras, bayesianos, classificadores de vizinho mais próximo, redes neurais artificiais e Support Vector Machine (SVM) [Witten et al., 2011]. 
VII Congresso Brasileiro de Informática na Educação (CBIE 2018)

Anais do XXIX Simpósio Brasileiro de Informática na Educação (SBIE 2018)

\subsection{Métricas de avaliação de classificadores}

Para distinguir entre a classe real e a classe prevista, são usados os rótulos (P - Positivo, NNegativo) para as previsões de classe produzidos por um modelo. Dado um classificador e uma instância a classificar, há quatro resultados possíveis:

- Se a instância é positiva e é classificada corretamente como positiva, ela é contada como um verdadeiro positivo (VP);

- Se a instância é positiva e é classificada incorretamente como negativa, é contada como um falso negativo (FN);

- Se a instância é negativa e é classificada corretamente como negativa, é contada como um verdadeiro negativo (VN); e

- Se a instância é negativa e é classificada incorretamente como positiva, é contada como um falso positivo (FP).

Dados um classificador e um conjunto de instâncias (o conjunto de teste), uma Matriz de Confusão de dimensões 2 × 2 pode ser construída representando as disposições do conjunto de instâncias para as duas classes. Esta matriz (Figura 01) forma a base para muitas métricas comuns de avaliação para os classificadores binários [Fawcett, 2006] [Silva et al., 2016].

Figura 01 - Matriz de Confusão para Classificadores.

\begin{tabular}{|c|c|c|c|}
\hline \multirow{2}{*}{$\pi \frac{0}{0}$} & & \multicolumn{2}{|c|}{ Classe Verdadeira (Referência } \\
\hline & & Negativo & Positivo \\
\hline \pm & Negativo & VN & $\mathbf{F N}$ \\
\hline $0 \leq$ & Positivo & FP & VP \\
\hline
\end{tabular}

Fonte: Adaptado de [Fawcett, 2006].

$\mathrm{Na}$ classificação binária, a classe de menor ocorrência é denotada como a classe positiva. A partir da Matriz de Confusão obtida após a classificação de todas as instâncias da base de testes, pode-se, então, obter as seguintes métricas que avaliam o poder preditivo do classificador [Fawcett, 2006] [Tan et al., 2009] [Silva et al., 2016]:

Acurácia (Accuracy) - Representa a taxa de acerto de todo o classificador e é obtida pela razão entre a soma dos acertos das duas classes e o número total de instâncias classificadas. É obtida pela expressão: Acurácia $=(\mathbf{V P}+\mathbf{V N}) /(\mathbf{V P}+\mathbf{V N}+\mathbf{F P}+\mathbf{F N})$. Obviamente, $\quad$ o melhor caso esperado para uma matriz de confusão é o preenchimento apenas da diagonal principal, o que resultaria em uma acurácia de $100 \%$.

Precisão (Precision) - Representa a preditividade positiva, que é o percentual de acertos de verdadeiros positivos dentre todos os exemplos classificados como positivos. Sua expressão é: Precisão = VP / (VP + FP). Quanto maior a precisão, menor o erro de falsos positivos cometidos pelo classificador.

Sensibilidade (Recall) - Indica a taxa de verdadeiros positivos, ou seja, o percentual de VP previstos corretamente. É obtida por Recall $=\mathbf{V P} /(\mathbf{V P}+\mathbf{F N})$. Um alto recall indica que o classificador produziu poucos exemplos positivos classificados como falso negativos.

\subsection{Trabalhos relacionados}

Alguns trabalhos recentes relataram técnicas de mineração de dados e aprendizagem de máquina para estabelecer perfis e realizar tarefas de predição da evasão na EAD. O trabalho de Paz e Cazella (2017) apresentou um estudo sobre o perfil dos estudantes de EAD com tendência à evasão, usando o classificador baseado em árvore de decisão (J48), alcançando uma acurácia de $90 \%$ nos resultados da classificação.

O estudo de Queiroga et al. (2017) relatou uma abordagem para detecção da possibilidade de evasão de estudantes de cursos técnicos a distância, a partir da contagem das interações dos estudantes no AVA e atributos relacionados. A geração e avaliação dos modelos 
VII Congresso Brasileiro de Informática na Educação (CBIE 2018)

Anais do XXIX Simpósio Brasileiro de Informática na Educação (SBIE 2018)

foi realizada por meio de cinco algoritmos (Bayes Net, Simple Logistic, Multilayer Perceptron, Random Forest e J48) e os resultados apontaram a possibilidade de predição de estudantes com risco de evasão ainda nas semanas iniciais dos cursos, com taxas de desempenho próximas a $75 \%$ na maioria dos cenários, e chegando a $95 \%$ nos melhores casos.

A pesquisa de Rigo et al. (2014) utilizou dados de disciplinas em três semestres de três cursos de graduação com o intuito de obter inferências em relação à evasão no curso. Os resultados da identificação de perfis de alunos evasores tiveram taxas de acerto na ordem de 87\%, utilizando o algoritmo RNA Multilayer Perceptron.

O trabalho de Dos Santos et al. (2014) utilizou dados de um curso superior completo nas suas análises, sem, entretanto, fundamentar a escolha dos dados usados no modelo preditivo. Os experimentos realizados a partir de algoritmos de árvores de decisão forneceram precisões médias de $81,55 \%$ utilizando as primeiras notas semestrais.

A carência de uma associação do processo de escolha das variáveis a uma teoria específica da EAD foi verificada nos trabalhos analisados. A definição das variáveis para mineração e definição dos modelos seguiu, na maioria dos casos, critérios estabelecidos pelos pesquisadores nos seus estudos.

\section{Percurso metodológico e materiais utilizados}

\subsection{Descoberta de Conhecimento em Banco de Dados}

A metodologia principal empregada neste estudo foi o instanciamento, para o contexto educacional, de um processo de descoberta de conhecimento em banco de dados (KDD Knowledge Discovery in Databases), que consiste na conversão de dados brutos em informações úteis. Esse processo é desenvolvido por meio de uma série de passos para transformação, do pré-processamento dos dados até o pós-processamento dos resultados da mineração de dados. A Figura 02 mostra, de maneira simplificada, o processo [Tan et al., 2009].

Figura 02- Processo de descoberta de conhecimento em banco de dados.

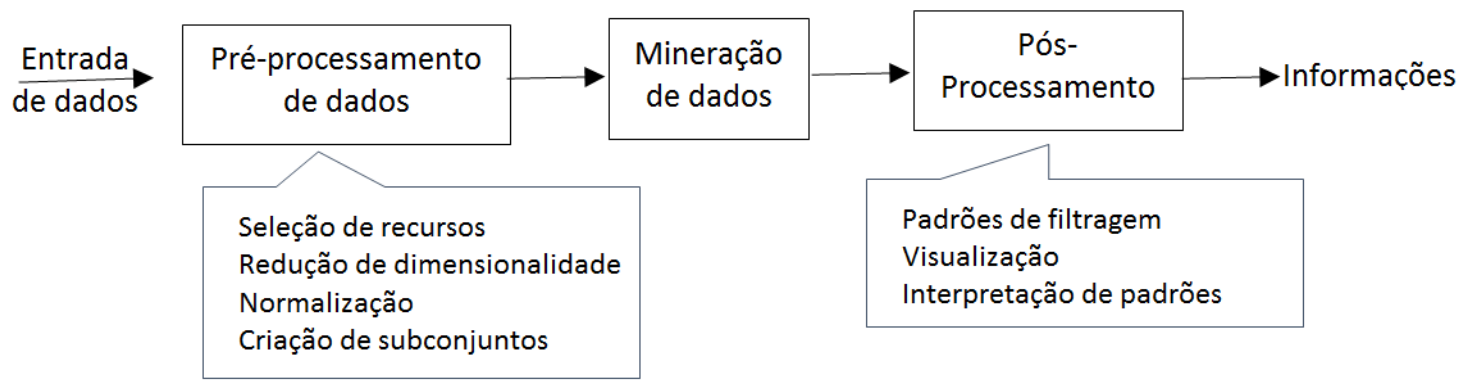

Fonte: [Tan et al., 2009]

Para Han et al. (2011) e Maimon e Rokach (2010), o KDD é uma análise exploratória, automática em grandes repositórios de dados. É o processo organizado de identificação de padrões válidos, novos, úteis e compreensíveis em conjuntos de dados grandes e complexos.

Alguns autores consideram a mineração de dados com o próprio KDD ou como seu sinônimo, como é o caso de Han et al. (2011) e Wang (2005), embora a maioria considere a mineração como sendo parte do processo de KDD. O trabalho clássico de Fayyad et al. (1996), estabeleceu bem os limites e diferenças de cada área. Para eles, o KDD refere-se a todo o processo de descoberta de conhecimento útil em dados e a mineração refere-se a uma determinada etapa nesse processo. A mineração de dados é a aplicação de algoritmos específicos para extrair padrões de dados.

Para a etapa inicial, na seleção e coleta das variáveis a serem usadas como entrada de dados no KDD, este estudo utilizou uma análise multivariada de dados, baseado no trabalho de Ramos et al. (2016), no qual, a partir da técnica de Análise Fatorial Confirmatória (AFC), 
VII Congresso Brasileiro de Informática na Educação (CBIE 2018)

Anais do XXIX Simpósio Brasileiro de Informática na Educação (SBIE 2018)

foram identificadas as variáveis mais relevantes que poderiam representar cada um dos três construtos (diálogo, estrutura e autonomia) da Teoria da Distância Transacional [Moore, 1993; 2013], em um banco de dados de um AVA, de uma Instituição de Ensino Superior (IES), na oferta regular de cursos de graduação por EAD. Esta teoria é considerada uma das mais relevantes para a EAD, sendo alvo de pesquisas e atualizações, não somente pelo seu autor, mas também por vários pesquisadores no mundo [Huang et al., 2015].

$\mathrm{Na}$ etapa seguinte, aconteceu a preparação dos dados que abrange todas as atividades necessárias para construir o conjunto de dados final, a partir dos dados brutos iniciais. A preparação de dados é um dos aspectos mais importantes e, muitas vezes, é o que exige mais tempo em mineração de dados, pois estima-se que essa etapa, geralmente, leva entre 50-70\% do tempo e esforço de um projeto [IBM, 2013]. Em um contexto de mineração de dados em um AVA, a preparação dos dados pode envolver, por exemplo, a limpeza dos dados nos logs de acessos dos alunos AVA.

$\mathrm{Na}$ etapa posterior ocorreu a mineração de dados, em busca de padrões de interesse em uma forma representacional particular ou um conjunto de tais representações, incluindo as regras de classificação. O processo de construção do modelo preditivo ocorre então por meio do ajuste de parâmetros realizado por um algoritmo e, quando o aprendizado de máquina é usado para esse fim, o processo é indutivo e comumente chamado de treinamento ou aprendizagem supervisionada. Após sua determinação, o modelo de predição pode, então, ser usado para prever rótulos em novos dados até então desconhecidos [Silva et al., 2016].

Quando o modelo determinado é aplicado em novos exemplares, tem-se a fase de teste, a partir da qual o modelo é avaliado quanto à sua capacidade preditiva. Muitas vezes, uma mesma base de dados é dividida em duas partes, sendo que uma delas usada para treinar e a outra para testar o modelo preditivo. Isso pode implicar processos de validações que garantam uma maior aleatoriedade na definição das duas bases.

A última etapa é a pós-processamento, onde acontece a avaliação e a interpretação dos padrões extraídos, podendo haver retorno a qualquer um dos passos anteriores para outra iteração. Esse passo também pode envolver a visualização dos padrões e modelos ou a visualização dos dados fornecidos pelos modelos extraídos. Nesse passo, o conhecimento descoberto também será documentado para posterior utilização.

\subsection{Bases de dados e tecnologias utilizadas}

A base de dados definida e obtida para ser explorada e analisada neste estudo, foi a de dois cursos de graduação por EAD da Universidade de Pernambuco (UPE): as licenciaturas em Pedagogia e em Ciências Biológicas. Esses dados foram oficialmente cedidos pela instituição, para fins de testes e validações. A escolha desses dois cursos foi em razão dos mesmos apresentarem os menores (Pedagogia, Turmas 1 e 2) e maiores (Biologia, Turmas 3 e 4) índices de evasão conforme informação da própria IES.

Os dados foram preparados e segmentados em duas bases diferentes para o processo de mineração: os dados de Pedagogia - Turma 1 e Biologia - Turma 3, por estarem completos com todos os períodos do curso, foram usados como bases para treinamento dos algoritmos e as outras duas foram usadas para base de testes dos modelos preditivos obtidos no processo. As bases distintas, resumidas na Tabela 01, treinaram e testaram a capacidade preditiva dos modelos, evitando problemas como sub e superestimação de parâmetros dos modelos.

Tabela 01 - Resumo das bases de treinamento e testes usados no estudo.

\begin{tabular}{|c|c|c|c|c|c|}
\hline $\begin{array}{c}\text { BASE DE } \\
\text { TREINAMENTO }\end{array}$ & $\begin{array}{l}\text { PERÍODOS } \\
\text { DO CURSO }\end{array}$ & $\begin{array}{c}\text { № } \\
\text { INSTÂNCIAS }\end{array}$ & BASE DE TESTES & $\begin{array}{l}\text { PERÍODOS } \\
\text { DO CURSO }\end{array}$ & $\begin{array}{c}\text { № } \\
\text { INSTÂNCIAS }\end{array}$ \\
\hline Biologia - Turma 3 & 8 & 1.150 & Biologia - Turma 4 & 6 & 3.190 \\
\hline Pedagogia - Turma 1 & 8 & 5.504 & Pedagogia - Turma 2 & 6 & 8.250 \\
\hline & TOTAL & 6.654 & & TOTAL & 11.440 \\
\hline
\end{tabular}

Fonte: Elaborado pelo Autor (2018). 
VII Congresso Brasileiro de Informática na Educação (CBIE 2018)

Anais do XXIX Simpósio Brasileiro de Informática na Educação (SBIE 2018)

Na operacionalização da pesquisa, diversos software foram utilizados, destacando-se: 1) SGBDs MySQL e PostgreSQL para extração e tratamento dos dados; 2) Microsoft Excel, para limpeza e preparação de tabelas, e 3) $R$ Project e RStudio, com diversos packages associados, para a análise multivariada, mineração de dados e aprendizagem supervisionada.

\section{Resultados e análises}

Os resultados são apresentados nessa seção em consonância com cada uma das etapas da metodologia usada, destacando, sempre que possível, aspectos mais relevantes destes resultados.

\subsection{Conjunto de variáveis utilizadas}

A partir da aplicação da análise fatorial confirmatória, conforme descrito em Ramos et al. (2016), foram identificadas a 21 (vinte e uma) variáveis mais relevantes que podem representar cada um dos três construtos da Teoria da Distância Transacional [Moore, 1993; 2013], na base de dados disponibilizada. Esse conjunto de variáveis é listado no Quadro 01.

Quadro 01 - Lista final de variáveis após a Análise Fatorial Confirmatória

\begin{tabular}{|l|c|}
\hline \multicolumn{1}{|c|}{ Variáveis } & Construto \\
\hline Média semanal da quantidade de acessos do aluno ao ambiente no semestre. & AUTONOMIA \\
\hline Quantidade de acessos do aluno ao ambiente por turno (Manhã), por semestre. & AUTONOMIA \\
\hline Quantidade de acessos do aluno ao ambiente por turno (Tarde), por semestre. & AUTONOMIA \\
\hline Quantidade de acessos do aluno ao ambiente por turno (Noite), por semestre. & AUTONOMIA \\
\hline Tempo médio semanal de utilização da plataforma pelo aluno no semestre. & AUTONOMIA \\
\hline Quantidade de acessos do aluno ao ambiente no semestre. & AUTONOMIA \\
\hline Qtd de acessos do aluno aos diferentes tipos de atividades, por disciplina. & AUTONOMIA \\
\hline Quantidade de acessos do aluno aos fóruns, por disciplina. & AUTONOMIA \\
\hline Quantidade geral de postagens do aluno em fóruns, por disciplina. & DIÁLOGO \\
\hline Quantidade geral de mensagens enviadas pelo aluno, por semestre. & DIÁLOGO \\
\hline Quantidade geral de mensagens recebidas pelo aluno, por semestre. & DIÁLOGO \\
\hline Qtd de colegas diferentes para quem o aluno enviou mensagens, por semestre. & DIÁLOGO \\
\hline Quantidade de mensagens dos professores recebidas pelo aluno, por semestre. & DIÁLOGO \\
\hline Quantidade de mensagens de colegas recebidas pelo aluno, por semestre. & DIÁLOGO \\
\hline Qtd de mensagens enviadas pelo aluno para outros colegas, por semestre. & DIÁLOGO \\
\hline Quantidade de mensagens enviadas pelo aluno aos professores, por semestre. & DIÁLOGO \\
\hline Quantidade geral de recursos disponibilizados pelo professor por disciplina. & ESTRUTURA \\
\hline Quantidade geral de atividades disponibilizadas pelo professor por disciplina. & ESTRUTURA \\
\hline Qtd de atividades com prazos de envio definidos por professor, por disciplina. & ESTRUTURA \\
\hline Qtd de fóruns de discussão disponibilizados sobre os conteúdos por disciplina. & ESTRUTURA \\
\hline Qtd de sessões de web conferências disponibilizadas no curso, por disciplina. & ESTRUTURA \\
\hline
\end{tabular}

Essas variáveis foram coletadas nas bases listadas na Tabela 01, formando dois conjuntos de dados com 6.654 instâncias para a base de treinamento e 11.440 instâncias para a base de testes.

Antes de serem analisadas as métricas de avaliação para cada classificador, é preciso observar como foram distribuídas, nas duas bases, as instâncias das duas classes analisadas: Evadiu e Não evadiu, para então serem feitas as inferências e análises adequadas. A Tabela 02 mostra a distribuição das instâncias nas duas bases coletadas.

Tabela 02 - № de casos (instâncias) de cada classe em ambas as bases.

\begin{tabular}{|c|c|c|c|c|c|}
\hline \multicolumn{3}{|c|}{ BASE TESTES } & \multicolumn{3}{|c|}{ BASE TREINAMENTO } \\
\hline Classe & № de Casos & $\%$ & Classe & № de Casos & $\%$ \\
\hline Não evadiu & 9.502 & $83,1 \%$ & Não evadiu & 4.941 & $74,3 \%$ \\
\hline Evadiu & 1.938 & $16,9 \%$ & Evadiu & 1.713 & $25,7 \%$ \\
\hline Total & 11.440 & $100 \%$ & Total & 6.654 & $100 \%$ \\
\hline
\end{tabular}

Fonte: Elaborado pelo Autor (2018).

A classe a ser considerada como "positiva" nas análises é geralmente a classe minoritária e também é a de maior interesse na pesquisa. Nesta pesquisa, a classe positiva foi a 
VII Congresso Brasileiro de Informática na Educação (CBIE 2018)

Anais do XXIX Simpósio Brasileiro de Informática na Educação (SBIE 2018)

"Evadiu". Ambas também representaram a variável alvo utilizada nos modelos dos classificadores.

\subsection{Resultados da aplicação dos Algoritmos de Classificação}

Para o processo de aprendizagem supervisionada, foram utilizadas as seguintes técnicas de classificação binária, com seus respectivos algoritmos: Árvore de Decisão (TreeDecision), Máquina de Vetor de Suporte (SVM), Rede Neural Artificial (NeuralNet), k-Nearest Neighbors (KNN) e Regressão Logística (RegLog).

A variável dependente do modelo foi o campo EVADIU presente nas bases, conforme mostrado na Tabela 2 no qual, para cada instância, foi atribuído o valor binário 0 (para não evadidos) e 1 (para evadidos). Essa rotulação serviu para que os fossem gerados os modelos preditivos na base de treinamento e que esses modelos pudessem ser avaliados, utilizando a base de testes.

Durante o processo de teste do modelo que foi gerado pelo classificador, usando os dados da base de treinamento, o sistema de aprendizado não prediz a classe, mas um valor contínuo ou ordinal que indica a probabilidade de cada instância analisada ser da classe "Evadiu" ou da "Não evadiu". Para se criar o modelo de classificação, esse valor deve ser binarizado pela escolha de um limiar de classificação.

Para a geração da matriz de confusão e cálculo das métricas do classificador, para cada instância, o valor da probabilidade foi convertido para uma variável dicotômica (binária), atribuindo-se o valor 0 para os não evadidos e 1 para os evadidos. Essa conversão se deu com o uso do valor 0,5 como limiar, no qual valores abaixo e até esse valor significavam baixa probabilidade de evasão. A partir daí o algoritmo fez a comparação entre o valor binário de referência (real) com o do modelo (previsto), produzindo os resultados a partir da matriz e respectivas métricas derivadas.

Foram geradas as matrizes de confusão, apresentando o resultado de cada modelo classificador, com os cálculos das principais métricas de avaliação para cada modelo: Precision, Recall e Accuracy. Além dessas métricas, foi também gerada a Curva ROC para cada um, com a determinação da área sob a curva (AUC). Esses quatro indicadores serviram de parâmetros para a determinação da técnica a ser adotada para o modelo preditivo final a ser implementado.

As matrizes de confusão dos classificadores usados na base completa de testes são apresentadas na Tabela 03. A diagonal principal indica o número de classificações corretas em cada classe (Verdadeiros Negativos e Verdadeiros Positivos) e a secundária, as classificações incorretas (Falsos Negativos e Falsos Positivos).

Tabela 03 - Matrizes de Confusão para cada classificador usando a base completa.

\begin{tabular}{|c|c|c|c|c|c|}
\hline Classificador & \multicolumn{2}{|c|}{ Referência (Real) } & RegLog & \multicolumn{2}{|c|}{ Referência (Real) } \\
\hline Modelo (Previsto) & NÃO EVADIU & EVADIU & Modelo (Previsto) & NÃO EVADIU & EVADIU \\
\hline NÃO EVADIU & VN & $\mathrm{FN}$ & NÃO EVADIU & 9035 & 742 \\
\hline EVADIU & FP & VP & EVADIU & 467 & 1196 \\
\hline NeuralNet & \multicolumn{2}{|c|}{ Referência (Real) } & KNN & \multicolumn{2}{|c|}{ Referência (Real) } \\
\hline Modelo (Previsto) & NÃO EVADIU & EVADIU & Modelo (Previsto) & NÃO EVADIU & EVADIU \\
\hline NÃO EVADIU & 8901 & 779 & NÃO EVADIU & 9146 & 837 \\
\hline EVADIU & 601 & 1159 & EVADIU & 356 & 1101 \\
\hline SVM & \multicolumn{2}{|c|}{ Referência (Real) } & TreeDecision & \multicolumn{2}{|c|}{ Referência (Real) } \\
\hline Modelo (Previsto) & NÃO EVADIU & EVADIU & Modelo (Previsto) & NÃO EVADIU & EVADIU \\
\hline NÃO EVADIU & 9314 & 1231 & NÃO EVADIU & 8807 & 754 \\
\hline EVADIU & 108 & 707 & EVADIU & 695 & 1184 \\
\hline
\end{tabular}

Fonte: Elaborado pelo Autor (2016).

Uma análise simplificada dessas matrizes pode ser feita tomando como exemplo o classificador baseado na Regressão logística. Dos 9.777 casos classificados como não evadidos pelo algoritmo, o modelo acertou 9.035 (92,4\%). Para os classificados como evadidos, o modelo 
VII Congresso Brasileiro de Informática na Educação (CBIE 2018)

Anais do XXIX Simpósio Brasileiro de Informática na Educação (SBIE 2018)

acertou 1.196 de 1.663 casos $(71,92 \%)$. As métricas de avaliação de cada classificador obtidas a partir das respectivas matrizes de confusão, são exibidas na Tabela 04 e seu respectivo gráfico comparativo na Figura 03.

\begin{tabular}{|c|c|c|c|c|c|}
\hline & RegLog & $S V M$ & NeuralNet & TreeDecision & $K N N$ \\
\hline RECALL & 0,617 & 0,365 & 0,598 & 0,612 & 0,568 \\
\hline PRECISION & 0,719 & 0,867 & 0,659 & 0,615 & 0,756 \\
\hline AUC & 0,856 & 0,796 & 0,828 & 0,788 & 0,797 \\
\hline ACCURACY & 0,894 & 0,882 & 0,879 & 0,869 & 0,896 \\
\hline
\end{tabular}

Fonte: Elaborado pelo Autor (2016).

O gráfico também mostra o valor de cada métrica nos cinco classificadores utilizados. Excetuando-se a SVM no Recall e Precision, os classificadores apresentaram valores próximos nas métricas. No geral, esses resultados foram bastante satisfatórios e duas das métricas indicaram um desempenho um pouco superior da Regressão Logística. A Acurácia (Acurácia = $(\boldsymbol{V P}+\boldsymbol{V N}) /(\boldsymbol{V P}+\boldsymbol{V N}+\boldsymbol{F P}+\boldsymbol{F N}))$ acima de 0,86 para todos os classificadores demonstra também uma boa taxa de acerto geral dos modelos na previsão.

Figura 03- Gráfico comparativo das métricas por classificador, base completa.

Comparativo Classificadores - Base Completa

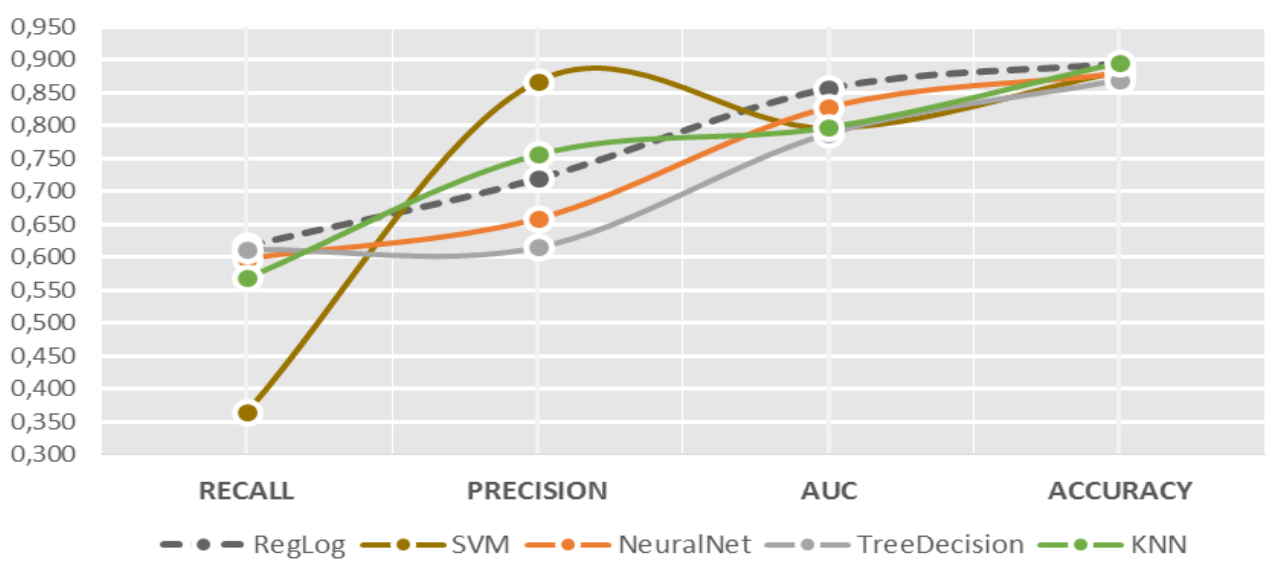

Fonte: Elaborado pelo Autor (2016).

A métrica de menor valor nos cinco classificadores é o Recall, ou seja, o percentual de verdadeiros positivos previstos corretamente pelo classificador $($ Recall $=V P /(V P+F N))$. Um valor alto no Recall indica que o classificador produziu poucos exemplos positivos erroneamente classificados como Falsos Negativos. Para os casos deste estudo, os Falsos Negativos representam os alunos evadidos, mas o modelo apontou o contrário. Os Falsos Positivos indicam os alunos que o modelo classificou como sendo evadidos, mas, na realidade, eles permaneceram no curso. Como é praticamente impossível gerar modelos perfeitos nos quais esses dois valores sejam zerados, busca-se então escolher qual técnica e respectivo algoritmo classificador que reduza o possível esses valores, além de analisar o desempenho sob outras métricas.

\subsection{Definição da Regressão Logística como classificador para evolução do estudo}

Embora as técnicas de Aprendizagem de Máquina/Mineração de Dados apresentadas e testadas no contexto desta pesquisa tenham, em diversas avaliações, apresentado resultados muito próximos, optou-se por prosseguir nas próximas etapas da pesquisa usando a Regressão Logística como a técnica responsável por gerar e testar os modelos preditivos, assim como sua futura implementação em um ambiente experimental para prova dos conceitos e alcance dos objetivos finais da pesquisa. 
VII Congresso Brasileiro de Informática na Educação (CBIE 2018)

Anais do XXIX Simpósio Brasileiro de Informática na Educação (SBIE 2018)

Além de apresentar índices melhores na maior parte das métricas, o uso da Regressão Logística, que estuda ou prevê o comportamento de uma variável dependente binária com base em um conjunto de variáveis independentes numéricas ou categóricas, pode ser justificado pelas seguintes considerações:

- A Regressão Logística, como técnica multivariada, é menos afetada quando suposições estatísticas básicas, como a normalidade dos dados, não são satisfeitas. Isso simplifica o processo inicial do KDD de entendimento e preparação dos dados;

- Esse método também pode acomodar diferentes tipos de variáveis independentes (métricas e não métricas). Os dados oriundos dos registros de banco de dados de AVAs são heterogêneos e podem ser de diferentes tipos, sem necessidade de processos adicionais de transformação nos dados;

- A técnica foi desenvolvida para prever a probabilidade de um valor binário ocorrer. Essa probabilidade pode ser transformada em eventos discretos e, com isso, possibilitar mudanças de limiares de transformação para binário e novas interpretações desses eventos;

- Os demais algoritmos de classificação analisados, embora também proporcionem altos níveis de precisão, são chamados de classificadores "caixa-preta", ou seja, eles não fornecem ao usuário todas as informações que levaram às previsões. Portanto, o conhecimento "por dentro" dos dados permanece oculto para o especialista e os usuários finais.

- A técnica já tem sua importância para a mineração de dados educacionais reconhecida pela comunidade de pesquisa da área, como na revisão do estado da arte feita por Peña-Ayala (2014), na qual para várias abordagens de EDM, a Regressão Logística foi a principal técnica usada nos trabalhos pesquisados. O trabalho de Marbouti et al. (2016) também reforça que essa é a técnica de previsão mais popular em contextos educacionais.

\section{Considerações finais}

A Educação a Distância representa um campo profícuo para pesquisadores em diversas áreas do conhecimento. Ao mesmo tempo que a modalidade é uma grande alternativa para formação e difusão do conhecimento, ainda são evidentes os seus obstáculos e desafios.

Um dos desafios que foi tratado neste estudo foi a questão da evasão na $\mathrm{EAD}$, que, no país, apresenta índices elevados e é ainda o principal desafio a superar, segundo grande parte das instituições de ensino superior que oferecem cursos na modalidade.

A redução das taxas de abandono é um dos pilares para a expansão e o sucesso deste tipo de curso. Um dos elementos-chave na redução das taxas de evasão é a identificação precisa e antecipada dos estudantes em situação de risco e o que pode estar provocando essa tendência. Assim que esses alunos forem identificados, os instrutores serão capazes de atender melhor às suas necessidades específicas e tomar as medidas apropriadas para reduzir sua probabilidade de abandonar o curso. Espera-se que a identificação prévia de alunos propensos à desistência também auxilie os gestores e instrutores em seus planejamentos estratégicos dos cursos

Está cada vez mais evidenciado que o uso de técnicas de mineração de dados em contextos educacionais oferece oportunidades para educadores e pesquisadores para obter mais conhecimentos úteis e relacionamentos mais interessantes entre as variáveis em grandes conjuntos de dados. Por meio das técnicas e algoritmos da mineração de dados, os pesquisadores podem descobrir quais comportamentos e tomar decisões que levam ao sucesso do aluno, identificar alunos que estão em risco de evasão ou de fraco desempenho, personalizar e adaptar o conteúdo e a instrução para atender às necessidades individuais e melhorar e otimizar o uso dos recursos educacionais. Além disso, considerando a apatia dos alunos em 
VII Congresso Brasileiro de Informática na Educação (CBIE 2018)

Anais do XXIX Simpósio Brasileiro de Informática na Educação (SBIE 2018)

relação às metodologias atuais de coleta de dados, como levantamentos baseados em questionários, pode ser mais eficaz e útil para pesquisadores educacionais coletarem dados abrangentes diretamente de AVAs.

É importante ressaltar que essas técnicas devem vir embutidas em aplicações computacionais de fácil uso, com interfaces amigáveis e com ferramentas de visualização, de modo a proporcionar aos usuários sem os conhecimentos específicos, maneiras transparentes de se beneficiarem das capacidades descritivas e preditivas da mineração de dados.

\section{Referências}

ABED. Censo EAD.BR: Relatório Analítico da Aprendizagem a Distância no Brasil - 2016. Associação Brasileira de Educação a Distância. 2017.

DOS SANTOS, R. et al. Uma Abordagem Genérica de Identificação Precoce de Estudantes com Risco de Evasão em um AVA utilizando Técnicas de Mineração de Dados. XIX Congresso Internacional de Informática Educativa. Fortaleza - CE 2014.

FAWCETT, T. An introduction to ROC analysis. Pattern recognition letters, v. 27, n. 8, p. 861-874, 2006. ISSN 0167-8655.

FAYYAD, U.; PIATETSKY-SHAPIRO, G.; SMYTH, P. From data mining to knowledge discovery in databases. AI magazine, v. 17, n. 3, p. 37, 1996. ISSN 0738-4602.

HAN, J.; KAMBER, M.; PEI, J. Data mining: concepts and techniques: concepts and techniques. Elsevier, 2011. ISBN 0123814804.

HUANG, X. et at. Understanding transactional distance in web-based learning environments: An empirical study. British Journal of Educational Technology 2015. ISSN 1467-8535.

IBM, C. IBM SPSS Modeler 16 User's Guide. p.50. 2013.

LANTZ, B. Machine learning with R. Packt Publishing Ltd, 2013. ISBN 1782162151.

MAIMON, O.; ROKACH, L. Data Mining and Knowledge Discovery Handbook. 2nd. Springer, 2010. ISBN 978-0-387-09822-7. Disponível em: < http://www.springer.com/br/book/9780387098227 >.

MARBOUTI, F.; DIEFES-DUX, H. A.; MADHAVAN, K. Models for early prediction of at-risk students in a course using standards-based grading. Computers \& Education, v. 103, p. 1-15, 2016.

MOORE, M. G. The theory of transactional distance. In: MOORE, M. G. (Ed.). Handbook of distance education. New York: Routledge 2013. cap. 5, p.66-85.

MOORE, M. G. Theory of transactional distance. In: (Ed.). Theoretical Principles of Distance Education. New York: Routledge, 1993. p. 2-29.

PAZ, F.; CAZELLA, S. Identificando o perfil de evasão de alunos de graduação através da Mineração de dados Educacionais: um estudo de caso de uma Universidade Comunitária. In: Anais dos Workshops do Congresso Brasileiro de Informática na Educação. 2017. p. 624.

PEÑA-AYALA, A. Educational data mining: A survey and a data mining-based analysis of recent works. Expert Systems with Applications, v. 41, n. 4, Part 1, p. 1432-1462, 2014a. ISSN 0957-4174. Disponível em: < http://www.sciencedirect.com/science/article/pii/S0957417413006635 >.

QUEIROGA, E.; CECHINEL, C.; ARAÚJO, R. Predição de estudantes com risco de evasão em cursos técnicos a distância. In: Simpósio Brasileiro de Informática na Educação-SBIE. 2017. p. 1547.

RAMOS, J. L. C., SILVA, J., RODRIGUES, R., GOMES, A. S., \& DE SOUZA, F. D. F. Mapeamento de dados de um LMS para medida de construtos da distância transacional. In: Simpósio Brasileiro de Informática na Educação-SBIE. 2016. p. 1056.

RIGO, S. J. et al. Aplicações de Mineração de Dados Educacionais e Learning Analytics com foco na evasão escolar: oportunidades e desafios. Revista Brasileira de Informática na Educação, v. 22, n. 01, p. 132, 2014.

SILVA, L. A.; PERES, S. M.; BOSCARIOLI, C. Introdução à Mineração de Dados - Com Aplicações em R. 1. Rio de Janeiro: Elsevier, 2016.

TAN, P.-N.; STEINBACH, M.; KUMAR, V. Introdução ao data mining: mineração de dados. Ciência Moderna, 2009. ISBN 8573937610.

WITTEN, I. H.; FRANK, E.; HALL, M. A. Data Mining: practical machine learning tools and techniques. Morgan Kaufmann, 2011. ISBN 008047702X. 